\title{
Estratégia de saúde da família (ESF), Núcleo de Apoio à Saúde da Família (NASF) e terapia ocupacional: problematizando as interfaces
}

\section{Family health strategy (ESF), Family Health Support Nuclei (NASF) \& occupational therapy: questioning the interfaces}

\author{
Selma Lancman', Juliana de Oliveira Barros ${ }^{2}$
}

\begin{abstract}
LANCMAN, S.; BARROS, J. O. Estratégia de saúde da família (ESF), Núcleo de Apoio à Saúde da Família (NASF) e terapia ocupacional: problematizando as interfaces. Rev. Ter. Ocup. Univ. São Paulo, v. 22, n. 3, p. 263-269, set./dez. 2011.

RESUMO: Os Núcleos de Apoio à Saúde da Família/NASF foram criados em 2008, pelo Ministério da Saúde, com vistas a apoiar as Equipes da Estratégia de Saúde da Família (EqSF) e, constituem-se hoje, num dos principais campos de trabalho para os terapeutas ocupacionais. Trabalham nos NASFs, profissionais de diferentes categorias, que devem atuar de forma compartilhada com as EqSF's a partir da estratégia do matriciamento. A implantação dos NASFs tem ocorrido de maneira irregular, sem capacitação apropriada, com má definição de papéis, com processos de trabalho por vezes conflituosos com as EqSF e sem contar com experiências já consolidadas que embasem as práticas. Contrasta ainda, demandas por ações de reabilitação, fruto da carência de serviços apropriados na rede de serviços de saúde. A partir de análise de documentos ministeriais, experiência prévia de trabalho de uma das autoras em equipe NASF e dos resultados preliminares de pesquisa sobre a temática, procurou-se contextualizar a implantação dos NASFs e refletir sobre a inserção e atuação dos terapeutas ocupacionais nessas equipes. A inserção e a definição do processo de trabalho dos vários profissionais e, dos terapeutas ocupacionais em particular, têm sido definidas através de documentos norteadores genéricos, que não especificam, não discriminam, nem dão suporte para as práticas cotidianas. $\mathrm{O}$ foco em ações de matriciamento previsto nos documentos norteadores conflita com as demandas da população por atendimentos específicos em nível de reabilitação, por exemplo, deixando os profissionais sobrecarregados e sem suporte para responder a essas demandas.
\end{abstract}

DESCRITORES: Saúde da família/recursos humanos; Atenção primária à saúde; Saúde do trabalhador; Terapia ocupacional.

\footnotetext{
1. Professora Titular do Departamento de Fisioterapia, Fonoaudiologia e Terapia Ocupacional da Faculdade de Medicina da USP. Coordenadora do Laboratório de Investigação e Intervenção em Saúde e Trabalho - LIIST / FMUSP.

2. Terapeuta Ocupacional do Laboratório de Investigação e Intervenção em Saúde e Trabalho do Departamento de Fisioterapia, Fonoaudiologia e Terapia Ocupacional da Faculdade de Medicina da USP. Mestre em Ciências da Reabilitação pela FMUSP.

Endereço para correspondência: Rua Cipotânea, no 51 - Cidade Universitária. Cep. 05360-160 - São Paulo, SP.
} 


\section{HISTÓRICO DAS POLÍTICAS PÚBLICAS E PRÁTICAS DE SAÚDE NA ATENÇÃO PRIMÁRIA}

$\mathrm{D}$ esde a criação do Sistema Único de Saúde SUS, pela Constituição de 1988, a promoção e a atenção à saúde vêm passando por transformações vinculadas, sobretudo, aos princípios e diretrizes que organizam o SUS e pelo reconhecimento da saúde como direito social (BRASIL, 1988).

Nesse cenário, o principio da integralidade se destaca visto que, o contexto social, o trabalho, a situação econômica, cultural, habitacional, relacional, e familiar das pessoas começam a ser considerados como determinantes das condições de saúde e de vida, ou seja, é ampliada a compreensão dos processos saúde-doença e do próprio conceito de saúde (BRASIL, 2009a).

Para ampliar o acesso aos serviços de saúde e concretizar o princípio da integralidade nas práticas de cuidado, o Brasil, em diálogo com as orientações da Organização Mundial da Saúde, reorientou o modelo assistencial a partir das ações desenvolvidas na atenção primária, com vistas à oferta de cuidados essenciais à saúde. Trata-se do primeiro nível de atenção que o usuário tem acesso para ingressar no SUS e deve estar acessível e próximo ao seu local de moradia e de trabalho. Tem como objetivo, organizar a rede de cuidados circunscrita a um território específico e viabilizar ao usuário a continuidade da atenção (OMS, 1978; CAPISTRANO FILHO, 1999; STARFIELD, 2002; BRASIL, 2006, 2009b).

As ações na atenção primária devem se dar nos âmbitos individual e coletivo de forma a privilegiar a participação social como um dos aspectos das práticas democráticas de cuidado e gestão. São dirigidas a territórios com áreas de abrangência delimitadas. Devem desenvolver ações de promoção, prevenção, diagnóstico, tratamento e reabilitação de forma integrada aos outros serviços da rede assistencial (OMS, 1978; BRASIL 2006, 2009b; STARFIELD, 2002; CAMPOS; AMARAL, 2007).

A aprovação da Política Nacional de Atenção Básica, no ano de 2006, marcou definitivamente a atenção primária em saúde como ponto de partida e porta de entrada preferencial do SUS. Definiu como prioridade: "consolidar e qualificar a estratégia Saúde da Família como modelo de Atenção Básica e centro ordenador das redes de atenção à saúde no SUS" (BRASIL, 2006).

A Estratégia de Saúde da Família - ESF, antes nomeada como Programa de Saúde da Família, criada em 1994, deu inicio ao processo de organização das práticas desenvolvidas na atenção primária (CAPISTRANO FILHO, 1999). Tem como base o trabalho em equipe, composta por médico, enfermeiro, auxiliar de enfermagem e agente comunitário de saúde, que se tornam referência para aproximadamente 1000 famílias, circunscritas no território específico da Unidade de Saúde da Família.

Estas equipes devem estar comprometidas em cuidar da saúde de famílias de forma humanizada, priorizando a constituição do vínculo de confiança, fundamental ao desenvolvimento do trabalho; fortalecer os processos de descentralização dos serviços e das ações de saúde; democratizar o acesso, a informação e a participação nos processos de construção da saúde; fomentar o direito a saúde como um direto de cidadania; trabalhar de forma integrada com a comunidade e fortalecer as ações intersetoriais com vistas à oferta de ações resolutivas.

No município de São Paulo, a ESF foi implantada em 1995, em algumas regiões, por meio de um convênio entre o Ministério da Saúde, a Secretaria Estadual de Saúde, a Casa de Saúde Santa Marcelina e a Fundação Zerbini, dando inicio a um modelo de gerenciamento baseado na parceria entre o público e as Organizações Sociais. Esta primeira experiência recebeu o nome de Qualidade Integral em Saúde - Qualis (CAPISTRANO FILHO, 1999; BOUSQUAT et al., 2006).

Contudo, nas demais regiões do município, o processo ocorreu de forma irregular e assistemática devido à implantação das cooperativas do Programa de Atenção à Saúde (PAS) que retardou a adesão municipal ao SUS. Assim, o início da municipalização da assistência à saúde só ocorreu em 2001, quando a ESF efetivamente se constituiu como estratégia organizadora do SUS em São Paulo. Em maio de 2010, já haviam 1.014 equipes de saúde da família (EqSF) cadastradas no município, oferecendo assistência a 3.494,850 habitantes (BOUSQUAT et al, 2006; BRASIL, 2010a).

Ainda no intuito de ampliar a abrangência e o escopo das ações da atenção primária e apoiar a inserção da ESF, em 2008 o Ministério da Saúde criou os Núcleos de Apoio à Saúde da Família - NASF (BRASIL, 2008; BRASIL, 2009b).

O NASF é composto por equipes de profissionais de diferentes categorias, entre eles o Terapeuta Ocupacional, previamente definidas pelos gestores locais segundo dados epidemiológicos. Essas equipes atuam de forma compartilhada com as EqSF's, nos territórios de sua responsabilidade a partir das demandas identificadas em conjunto. Diferentemente da EqSF, o NASF não é porta de entrada para o SUS e configura-se como equipe de apoio 
(BRASIL, 2008, 2009b).

Nesse contexto, tendo em vista a possibilidade de inovação das práticas de cuidado e da inserção profissional do Terapeuta Ocupacional trazidas pela proposta do NASF, este artigo tem como objetivo apresentar e discutir possíveis contradições e desafios enfrentados na implantação deste modelo além de discutir a inserção do Terapeuta Ocupacional no campo.

Para tanto, foram analisadas: as publicações do Ministério da Saúde sobre ESF e NASF; a experiência profissional de uma das autoras na composição de equipe de NASF, no período de novembro de 2008 a dezembro de 2010 e os resultados preliminares da pesquisa intitulada "O Processo de Trabalho Nos Núcleos de Apoio à Saúde da Família (NASF) e seus Efeitos na Saúde Mental dos Trabalhadores", que está em andamento. Tal pesquisa foi financiada pelo Conselho Nacional de Desenvolvimento Científico e Tecnológico (CNPq - Edital Universal 2010) e também foi aprovada pelo Comitê de Ética da Faculdade de Medicina da Universidade de São Paulo sob protocolo número 160/11 e pelo Comitê de Ética da Prefeitura Municipal de São Paulo, sob protocolo número 89/11.

\section{APRESENTANDO O NASF}

De forma geral, o NASF deve buscar, junto às EqSF's, a concretização do cuidado integral, a partir da qualificação e complementaridade das ações de promoção, prevenção, assistência e reabilitação em saúde e a organização e coordenação da rede de cuidados, com vistas ao acompanhamento longitudinal dos usuários, aumentando a resolutividade e diminuindo os encaminhamentos a outros níveis de atenção (BRASIL, 2008, 2009b).

Estão organizados em duas modalidades: NASF $1 \mathrm{e}$ NASF 2. O NASF 1 pode ser composto por equipes de no mínimo 5 profissionais, que apoiam entre 8 e 20 EqSF's, exceto nos estados da Região Norte, onde o número mínimo de EsSF's passa a ser 5. Já o NASF 2 é composto por no mínimo 3 profissionais, não sendo prevista a presença de médicos e está vinculado a no mínimo 3 EqSF's. Em decorrência da magnitude epidemiológica dos transtornos mentais recomenda-se que cada equipe contemple ao menos 1 profissional desta área (BRASIL, 2008).

Entre as atribuições dos profissionais do NASF estão: conhecer e articular os serviços existentes no território promovendo a intersetorialidade; conhecer a realidade das famílias residentes na área adscrita, nos seus aspectos econômico, social, cultural, demográfico e epidemiológico; identificar, em conjunto com a comunidade e a EqSF, o público prioritário para o desenvolvimento das ações e a abordagem a ser adotada; prevenir e promover a saúde por meio de ações educativas; promover ações interdisciplinares junto as EqSF's, por meio de discussões periódicas, visando uma abordagem coletiva (BRASIL, 2008, 2009b).

As áreas estratégicas para atuação do NASF estão organizadas em torno de nove temáticas: saúde da criança/ do adolescente e do jovem; saúde mental; reabilitação/ saúde integral da pessoa idosa; alimentação e nutrição; serviço social; saúde da mulher; assistência farmacêutica; atividade física/ práticas corporais; práticas integrativas e complementares (BRASIL, 2009b).

A principal metodologia de trabalho utilizada pela equipe NASF é o matriciamento, que tem como objetivo assegurar retaguarda especializada às EqSF's. Este apoio pode acontecer de duas formas: a primeira se dá a partir da oferta de assistência especializada ao usuário nas situações de maior complexidade, após pactuação entre EqSF e NASF; e a segunda se dá pela oferta de suporte técnico pedagógico. Esse suporte ocorre, prioritariamente, de duas maneiras: a partir do desenvolvimento de intervenções compartilhadas entre profissionais do NASF e da EqSF atendimentos na unidade, visitas domiciliares, grupos na unidade de saúde ou na comunidade - e a partir da troca de informações e orientações entre eles, seja sobre um caso especifico, seja sobre uma problemática apresentada pela EqSF (CAMPOS; DOMITTI, 2007; BRASIL, 2008, 2009b).

As abordagens são acordadas em reuniões com a presença de profissionais das duas equipes. A freqüência destes encontros é negociada entre as equipes onde as EqSF apresentam casos considerados mais complexos e que necessitam de apoio especializado (BRASIL, 2008).

Os resultados esperados estão relacionados à ampliação do trabalho e a melhor resolutividade das situações de maior complexidade já desenvolvido pela EqSF. Espera-se ainda, diminuir a fragmentação dos processos de trabalho e do cuidado oferecido (BRASIL, 2008, 2009b).

\section{Os Processos de Trabalho na ESF e no NASF: comple-} mentaridades e contradições

Devido ao tempo de implantação, a ESF tem estratégias de ação e processos de trabalho mais 
consolidados, incluindo documentos norteadores, que discriminam as atribuições específicas de cada profissional e o tempo que deve ser utilizado em cada ação. Já os documentos referentes ao NASF, apresentam orientações genéricas das ações a serem desenvolvidas sem discriminar com clareza os processos de trabalho a serem empregados. Essas diferenças nas atribuições das duas equipes podem estar criando processos de trabalho conflituosos e até mesmo contraditórios.

A utilização da metodologia do apoio matricial pretende ampliar a compreensão sobre o processo saúdedoença a partir da escuta e integração de diversos saberes e práticas, favorecendo, inclusive, a operacionalização de um paradigma que compreenda estes processos de forma mais complexa. Contudo, a implementação desta prática depende de uma nova postura profissional, pautada pela ética do cuidado e do compromisso com os princípios e diretrizes do SUS (BRASIL, 2009b).

A metodologia do apoio matricial está prevista desde o inicio das ações do projeto Qualis e é a base para o desenvolvimento do trabalho do NASF. Contudo, trata-se de uma proposta inovadora no campo da saúde e sua implantação varia de região para região e não conta com processos de trabalho definidos e sistematizados. Desta forma é possível que, no cotidiano das práticas, os profissionais se deparem com dificuldades distintas para a operacionalização da proposta (CAMPOS; DOMITTI, 2006).

A primeira dificuldade, de âmbito estrutural, se refere às diferentes formas de organização do trabalho da EqSF e do NASF que, no entanto, devem atuar de forma compartilhada (PMSP/SMS, 2009). Enquanto no NASF está previsto que os trabalhadores destinem a maior porcentagem das suas horas de trabalho em ações tais como, reuniões, consultas, visitas domiciliares e grupos compartilhados com EqSF, os trabalhadores da EqSF, sobretudo os profissionais de nível superior, têm a maior parte das suas horas de trabalho voltadas para o desenvolvimento de um elevado número de consultas individuais. A título de exemplo, o médico, para dar conta da meta prevista pelo programa, de 400 consultas mensais, precisa realizar consultas individuais de, em média, 15 minutos (PMSP/SMS, 2005, 2009; CAMPOS; DOMITTI, 2006).

A segunda dificuldade, que interfere no trabalho das duas equipes, decorre da inter-relação entre o excesso de demanda e a carência de recursos. Pela precariedade da rede assistencial e dificuldade de encaminhar casos de maior gravidade, os trabalhadores do NASF podem sentir-se pressionados pela população, pelas EqSF's e pelas demandas que presenciam, a desenvolverem ações de forma ambulatorial, especializadas e individualizadas. A utilização do apoio matricial tem como potência a qualificação e identificação das prioridades de atendimento especializado, contudo, na ausência de uma rede de suporte que assegure a continuidade dos atendimentos em níveis de maior complexidade corre-se o risco de se ceder a essas pressões.

A terceira dificuldade diz respeito à presença de Organizações Sociais na gestão de serviços públicos, processo validado no âmbito Federal e largamente implementado em São Paulo (BRASIL, 1998). Essa forma de gestão termina mesclando o caráter público e privado dos serviços oferecidos, criando situações de trabalho heterogêneas a depender da parceira envolvida. Essa heterogeneidade de praticas profissionais nas diferentes regiões da cidade pode dificultar a criação de uma cultura comum entre as várias equipes comprometendo a troca de experiências e a consolidação das políticas públicas.

Soma-se a isso a precarização dos contratos de trabalho dos profissionais vinculados a estas instituições parceiras e a falta de padronização dos processos seletivos, salários e condições de trabalho.

A quarta dificuldade diz respeito à existência de um documento norteador que define de forma frágil as atribuições dos diversos profissionais que compõem as equipes do NASF. A pouca prescrição do trabalho aliada a falta de uma cultura de trabalho interdisciplinar e de uma experiência acumulada nesse tipo de serviço, favorece que cada segmento atue isoladamente, que aja segundo seu lugar específico de saber, advindo dos processos de formação profissional compartimentalizado. Essa situação propicia ainda atuações arbitrárias advindas de diferentes compreensões do processo saúde-doença, tais como, ações voltadas para aspectos orgânicos dicotomizadas dos aspectos psíquicos e/ou sociais; ações de caráter mais curativo em detrimento de ações de promoção a saúde.

Além disso, ou justamente em função do exposto, tudo indica que pode ocorrer nas equipes do NASF uma certa indiscriminação do perfil da clientela alvo, dos objetivos principais do trabalho, cabível para as equipes, para cada um dos profissionais e para os diferentes serviços. Da mesma forma, é de se esperar que uma prescrição e trabalho tão vaga deixe os vários profissionais, e o terapeuta ocupacional em particular, em conflito sobre o trabalho a ser realizado, e das prioridades e especificidades de sua ação. 


\section{Inserção do Terapeuta Ocupacional no NASF: proble- matizando um novo perfil profissional}

Em maio de 2010 existiam no país, 1165 NASF's em funcionamento, abarcando 6895 trabalhadores. A meta até o final do ano de 2011 é de que 1500 NASF's estejam implantados. Em relação a São Paulo, dados referentes mesmo período mostram que existiam 48 NASF's do tipo II em funcionamento (BRASIL, 2010b,c).

Ainda em relação ao mesmo período, no país existiam aproximadamente 300 Terapeutas Ocupacionais inseridos nos NASF's. Trata-se, portanto, de um dos principais campos de trabalho para esses profissionais (BRASIL, 2010b).

Desde a implantação do projeto QUALIS, o Terapeuta Ocupacional já atua na atenção primária em saúde, a partir de duas grandes áreas: Reabilitação e Saúde Mental. Contudo, a proposta do NASF traz a necessidade de um profissional generalista, afinal todos os profissionais do NASF devem apoiar as EqSF's em demandas distintas que sejam relevantes nos territórios específicos de ação: desde saúde da mulher, gestante, criança, até a saúde dos idosos, questões de violências, entre outras.

Nesse contexto, qual é a contribuição do Terapeuta Ocupacional? Como pensar e desenvolver suas práticas neste nível de atenção?

De forma geral, o cotidiano, o trabalho no território e o fomento dos processos de inclusão social são compreendidos enquanto objetos de ação dos Terapeutas Ocupacionais na Atenção Primária e, conseqüentemente daqueles profissionais inseridos em NASF's (JARDIM et al., 2008).

Entretanto, diante de atribuições tão semelhantes com as demais profissionais e com a fragilidade de prescrição contida nos documentos norteadores, como discriminar a especificidade do trabalho do Terapeuta Ocupacional e afirmar seu lugar? Ou ainda, diante das carências de serviços no âmbito da atenção secundária e terciária, o que dificulta o acesso da população a estes níveis de atenção, como direcionar seu trabalho? E, nesse caso, como oferecer um atendimento especializado sem os recursos apropriados?

Os terapeutas ocupacionais vivem assim o seguinte dilema: direcionar seu trabalho para ações de promoção a saúde e desenvolver estratégias de matriciamento visando a construção de projetos coletivos com as EqSF ou, realizar atendimentos individuais de casos considerados graves. Se, as atividades de matriciamento são consideradas como as principais e aquelas que garantirão, a médio prazo, mudanças mais efetivas nas práticas assistenciais, as necessidades prementes de pessoas por atendimento individual, na ausência de serviços secundários ou terciários na rede de atendimento, leva esses profissionais a terem que dividir seu tempo e atenção. Destaca-se, ainda, que os profissionais, não dispõem de recursos materiais para desenvolver praticas de reabilitação que, nesse caso, acontecerão necessariamente de forma precária e improvisada.

O pouco tempo de existência do NASF, a novidade dessa forma de atuação para os terapeutas ocupacionais, a falta de práticas consolidadas onde possam se espelhar e a pressão por atendimento individual de populações desassistidas em outros níveis de atenção, deixa esses profissionais sem respaldo e mal aparelhados no seu cotidiano de trabalho, sem parceiros nem recursos para criarem uma prática em terapia ocupacional que faça avançar a sua atuação nesse importante projeto nacional.

\section{CONSIDERAÇÕES FINAIS}

Apesar de existirem diversos estudos sobre o processo de trabalho na ESF (LANCMAN et al., 2007; ALONSO, 2009; JARDIM; LANCMAN, 2009), a produção sobre o trabalho do NASF ainda é escassa, provavelmente por se tratar de uma proposta recente e estar ainda em fase de implantação.

A implantação dos NASF tem acontecido de maneira irregular, sem processos de capacitação apropriados, com má definição de papéis, com processos de trabalho por vezes conflituosos com as EqSF's de quem necessitam ser parceiros e sem contar com experiências já consolidadas que embasem as práticas profissionais. Não é de se estranhar que as equipes se sintam sobrecarregadas e mal preparadas para responder as varias demandas apresentadas pelos usuários, pelas EqSF's e pelas carências do sistema de saúde.

Trata-se de um importante campo de trabalho para os Terapeutas Ocupacionais, que, ao longo de sua história já trazem experiências significativas na Atenção Primária em Saúde. Fica, então, o desafio de fazer a interlocução da proposta apresentada nos documentos norteadores do NASF com os distintos interesses e situações reais que se colocam nas vivências práticas do trabalho para os profissionais. 
LANCMAN, S.; BARROS, J. O. Family Health Strategy (ESF), Family Health Support Nuclei (NASF) \& Occupational Therapy: questioning the interfaces. Rev. Ter. Ocup. Univ. São Paulo, v. 22, n. 3, p. 263-269, set./dez. 2011.

\begin{abstract}
The Family Healthcare Support Nuclei/NASF were created in 2008 by the Ministry of the Health to support the Family Healthcare Strategy Teams (EqSF) and today they are one of the main fields of work for occupational therapists. Different healthcare providers work at the NASFs, who are supposed to share the work with the EqSFs based on the strategy of interconnected basic (preventive care/public education), secondary (diagnosis/treatment) and tertiary (healing/ rehabilitation) public healthcare services. The NASFs have been irregularly implemented, without adequate qualification processes, with a bad definition of roles and work processes sometimes conflicting with those of the EqSFs, without relying on consolidated experiences where to base their practices on. Some demands for rehabilitation cannot be met due to shortage of adequate services in the healthcare network. Starting from a bibliographical review mainly based on the Ministry of the Health's documents, a previous work experience of one of the authors in NASF teams, and on preliminary results of a research on the theme, this article contextualizes the NASFs implementation and reflects upon the insertion and performance of occupational therapists in those teams. Inserting and defining the work processes of different healthcare providers, particularly those of occupational therapists, have been based on documents with generic guidelines that fail to specify, discriminate or support daily practices. The focus on actions targeted on interconnecting basic, secondary and tertiary public healthcare services disposed in those guidelines conflicts with the population's demands for specific rehabilitation care, among others, which results in overloaded and unsupported professionals unable to meet such demands. Descriptors: Family healthcare support nuclei, basic healthcare services, work process in healthcare, occupational therapy.
\end{abstract}

KEY WORDS: Family health/manpower; Primary health care; Occupational health; Occupational therapy.

\section{REFERÊNCIAS}

ALONSO, C. O trabalho e o trabalhador de uma equipe de reabilitação no Programa Saúde da Família do município de São Paulo. Dissertação (Mestrado Medicina) - Faculdade de Medicina, Universidade de São Paulo. São Paulo, 2009.

BOUSQUAT, A.; COHN, A.; ELIAS, P. A. Implantação do Programa Saúde da Família e exclusão sócio - espacial no município de São Paulo, Brasil. Cad. Saúde Pública, Rio de Janeiro, v. 22, n. 9, p. 1935-1943, 2006.

BRASIL. Constituição da República Federativa do Brasil. Brasília, DF: Imprensa Oficial, 1988.

BRASIL. Ministério da Administração Federal e Reforma do Estado / Secretaria da Reforma do Estado. Organizações sociais. 5a. ed. Brasília: Ministério da Administração e Reforma do Estado, 1998. v.2 (Cadernos MARE da reforma do estado) [Acesso em 20 jun. 2010]. Disponível em: <http://www.mp.gov.br/secretarias/ upload/Arquivos/publicacao/seges/PUB_Seges_Mare_caderno02. PDF>

BRASIL. Ministério da Saúde. Secretaria de Atenção à Saúde. Departamento de Atenção Básica. Política nacional de atenção básica. Brasília : Ministério da Saúde, 2006.
BRASIL. Ministério da Saúde. Portaria 154 de 24 de janeiro de 2008. Cria os Núcleos de Apoio à Saúde da Família - NASF. Brasília; 2008 [Acesso em 20 jun. 2010]. Disponível em: $<$ http://200.137.177.147/sistemas_de_informacao/doc_tec_leg/ siab/portaria-n-154-nasf.pdf $>$.

BRASIL. Ministério da Saúde. Conselho Nacional das Secretarias Municipais de Saúde. O SUS de A a Z : garantindo saúde nos municípios. 3a. ed. Brasília: Editora do Ministério da Saúde; 2009a [Acesso em 23 jun. 2010]. Disponível em: <http://portal.saude. gov.br/portal/arquivos/pdf/sus_3edicao_completo.pdf $>$.

BRASIL. Ministério da Saúde. Secretaria de Atenção à Saúde. Departamento de Atenção Básica. Diretrizes do NASF - Núcleo de Apoio à Saúde da Família. Brasília: Ministério da Saúde; 2009b. (Série B. Textos Básicos de Saúde / Cadernos de Atenção Básica; n. 27).

BRASIL. Ministério da Saúde. Secretaria de Atenção à Saúde. Departamento de Atenção Básica. Teto, credenciamento e implantação das estratégias de Agentes Comunitários de Saúde, Saúde da Família e Saúde Bucal. Unidade geográfica: Município de São Paulo. Competência: jan. 1998 / mai. 2010. 2010a [Acesso em 23 jun. 2010]. Disponível em: <http://dab.saude.gov.br/ 
historico_cobertura_sf/historico_cobertura_sf_relatorio.php $>$

BRASIL. Ministério da Saúde, Secretaria de Atenção à Saúde. Cadastro Nacional de Estabelecimentos de Saúde (CNES). 2010b [Acesso em 01 jul. 2010]. Disponível em: $<$ http://cnes.datasus. gov.br/Mod_Ind_Equipes.asp?VEstado $=35 \&$ VMun $=00 \&$ VCom $\mathrm{p}=201005->$

BRASIL. Ministério da Saúde. Secretaria de Atenção à Saúde. Departamento de ações programáticas estratégicas. Coordenação geral de saúde mental, álcool e outras drogas. Saúde Mental Dados 7, Brasilia, ed. esp., n. 7, 2010c.

CAMPOS, G. W. S.; AMARAL, M. A. A clínica ampliada e compartilhada, a gestão democrática e redes de atenção como referenciais teórico-operacionais para a reforma do hospital. Ciênc. Saúde Coletiva, v. 12, n. 4, p. 849-859, 2007.

CAMPOS, G. W.; DOMITTI, A. C. Apoio Matricial e equipe de referência: uma metodologia para gestão do trabalho interdisciplinar em saúde. Cad. Saúde Pública, Rio de Janeiro, v. 23, n.2, p. 399-407; 2007.

CAPISTRANO FILHO, D. O programa de saúde da família em São Paulo. Estudos Avançados, v. 13, n. 35, 1999 [Acesso em 25 jun. 2010]. Disponível em: <http://www.scielo.br/scielo. php?pid=S0103-40141999000100008\&script=sci_arttext $>$

JARDIM. T.A.; AFONSO, V. C.; PIRES, I. C. A terapia ocupacional na Estratégia de Saúde da Família - evidências de um estudo de caso no município de São Paulo. Rev. Ter. Ocup. Univ. São Paulo, v. 19, n. 3 , p. $167-175,2008$.

JARDIM, T. A.; LANCMAN, S. Aspectos subjetivos do morar e trabalhar na mesma comunidade: a realidade vivenciada pelos agentes comunitários de saúde. Interface. Comun. Saúde Educ., v. 13, p. 123-135, 2009.

FERNANDES, C. C.; LAURENTINO, D. A. P.; LIMA, L. J. C. Desafios e conquistas da atuação da terapia ocupacional no núcleo de apoio a saúde da familia na região central de são paulo-sé. In: CONGRESSO LATINO-AMERICANO DE TERAPIA OCUPACIONAL, 9., São Paulo, 2011. Anais eletrônicos. Disponível em: <http://www.cbtoeclato2011.com. $\mathrm{br} / \mathrm{cd} /$ listaresumos.htm>

LANCMAN, S.; UCHIDA, S.; SZNELWAR, L.; JARDIM, T. A.; GASPARO, S. O trabalhar no programa saúde da família: um estudo em psicodinâmica do trabalho. In: INQUÉRITO com usuários e profissionais, percepção dos gestores e estudos sobre o trabalho no PSF; Cadernos de Atenção Básica, estudos avaliativos 3. São Paulo: Consorcio Medicina USP/ Centro de Estudos de Cultura Contemporânea, 2007. v. 3, p. 207-228.

MATSUKURA, T. S.; CARVALHO, V. C. Identificando práticas de terapeutas ocupacionais inseridos no contexto da atenção básica em saúde. In: CONGRESSO LATINO-AMERICANO DE TERAPIA OCUPACIONAL, 9., São Paulo, 2011. Anais eletrônicos. Disponível em: <http://www.cbtoeclato2011.com. $\mathrm{br} / \mathrm{cd} /$ listaresumos.htm>.

OMS. Declaração de Alma - Ata. Conferência Internacional sobre cuidados primários de saúde. Alma-Ata; 1978 [Acesso em 20 jun. 2010]. Disponível em: <http://www.opas.org.br/coletiva/ uploadArq/Alma-Ata.pdf $>$.

SÃO PAULO. Prefeitura do Município de São Paulo, Secretaria Municipal de Saúde. Atenção Básica e PSF. Documento norteador: compromisso das Unidades Básicas de Saúde com a População. São Paulo, 2005.

PAIVA, L. F. A.; SOUZA, F. R.; SAVOLI, K. C.; VIEIRA, J. L. A terapia ocupacional na atenção básica: a construção de uma nova práxis. In: CONGRESSO LATINO-AMERICANO DE TERAPIA OCUPACIONAL, 9., São Paulo, 2011. Anais eletrônicos. Disponível em: <http://www.cbtoeclato2011.com. $\mathrm{br} / \mathrm{cd} /$ listaresumos.htm>

SÃO PAULO. Prefeitura do Município de São Paulo, Secretaria Municipal de Saúde. Coordenação da Atenção Básica. Estratégia Saúde da Família. Diretrizes e parâmetros norteadores das ações dos Núcleos de Apoio à Saúde da Família (NASF). São Paulo, 2009 .

STARFIELD, B. Atenção primária: equilibrio entre necessidades de saúde, serviços e tecnologia. Brasília: Unesco, Ministério da Saúde, 2002. 